\title{
Automatic Configuration Method of Smart Substation Process Layer Network
}

\author{
Qing Yang, Hui Ren, Guoqing Zhao and Haidong Zhang \\ China Electric Power Research Institute (Nanjing), Nanjing 210003, China
}

\begin{abstract}
This paper analyzes the problems in the process network configuration of smart substation. A method for automatic configuration of process layer network in smart substation based on SDN is proposed. First, establish a physical port model that defines the configuration information for the process layer network. Secondly, the physical port model is parsed and processed to generate flow tables and group tables in SDN technology. The automatic configuration method of the process layer network is realized by the unified configuration of the SDN controller to the switch. Finally, the simulation case is built on the mininet simulation platform, and the correctness and feasibility of the method is verified.
\end{abstract}

Keywords-smart substation; process level; network configuration; physical port model; SDN

\section{INTRODUCTION}

In the smart substation, because the process layer network needs to transmit and interactively sample data, control the data, its importance has been no less than the protection control device itself ${ }^{[1-3]}$ In order to make the process layer network packets meet the real-time and reliability requirements, reduce the network congestion and network storm, usually use the network switch to divide the VLAN.

At present, VLAN technology is the most widely used packet filtering technology in smart substation, and the process layer network is usually divided into VLAN according to the switch port, which does not involve the connected intelligent electronic devices. But the switch VLAN configuration, usually use the "one to one" mode, cannot be unified configuration, the repetitive work not only increases the debugging workload, but also makes the debugging personnel brain fatigue, human error is not necessary, work efficiency is reduced. At the same time, different manufacturers of switches, VLAN configuration methods are not the same, which requires the debugger to understand each vendor's configuration method, the configuration work to bring a certain degree of difficulty ${ }^{[4-6]}$.

In order to solve the above problems, literature [7] proposed a process layer network automatic configuration method, the method is the use of offline simulation of the way to generate a static configuration file, and then download the configuration file to the switch. However, this method requires different configuration files for switches from different manufacturers.

Software defined network (SDN) is a new type of network architecture, the basic idea is to control the phase separation and forwarding, i.e. SDN switch only has the functions of data forwarding, forwarding strategy management by the unified controller, so as to realize the unified configuration. At the same time, the SDN switches and controllers communicate with each other through a unified southbound interface, such as the OpenFlow protocol. Therefore, the problem of different configuration methods for different manufacturers' switches can be solved ${ }^{[8-10]}$. Based on SDN technology, this paper proposes a method for automatic configuration of process layer network. Construction of physical port model, a model describing the process layer network configuration information, will work to build configuration model of the network, through the analysis of the physical port model, automatic SDN control strategy is issued to the SDN switch, complete network configuration. This method not only solves the problem of the unified configuration of different manufacturers' switches, but also improves the work efficiency.

\section{SOFTwARE Defined NeTWORK}

Software defined network is a new network architecture and technology that can be programmed by software. It has the characteristics of separation of control and forwarding, centralization of network control, and flexible programming ability of software. The software definition network architecture includes three layers, from top to bottom, application layer, control layer and data layer. Application layer is composed of a variety of business applications, users can write applications according to requirements. The control layer is mainly used to manage network topology and related resource information, and to formulate and transmit data forwarding strategy is the core control part of the network. Data layer is usually composed of SDN switches, the main task is data processing, forwarding and status acquisition. In addition, the northbound interface between application layer and control layer, the southward interface between control layer and data layer, is also an important component of the software defined network. As shown in the following figure:

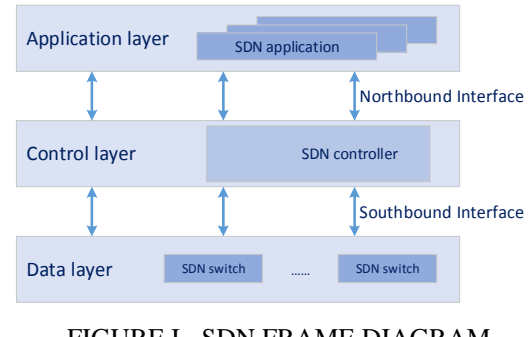

OpenFlow protocol is one of the standard protocols of SDN southbound interface. It specifies the communication rules 
between OpenFlow switches and controllers, so that network devices have programmable capabilities. Based on Open Flow SDN is based on the flow table mechanism to achieve. The socalled flow table, in fact, can be seen as OpenFlow network device data forwarding function of an abstract, which integrates the network at all levels of network configuration information, is responsible for data packet query and forwarding ${ }^{[11]}$. There is only one group table in the OpenFlow switch that contains multiple group table entries. Each group table item can contain a lot of action buckets. The group type specifies the method of execution of instructions in the action bucket, in which the type "ALL" represents the execution of actions in all action buckets, applicable to broadcast or multicast situations. Therefore, the transmission of GOOSE and SV messages in the process layer network can be accomplished by setting up a group table.

\section{Automatic CONFiguration METHOD}

Aiming at the problem of network configuration in process layer, a method of automatic configuration for process layer network in smart substation is proposed. By establishing the physical port model of the device, the process layer network configuration is embodied in the physical port model, analysis the physical port model, the formation of data structure, and finally generate flow table and set of tables in SDN technology, and issued to the process layer switch (SDN switch), can realize the automatic configuration of the process layer network, but also to achieve a unified configuration process layer network switch.

Process layer network automatic configuration implementation method is divided into 3 steps:

\section{A. Modeling of Device Physical Port Model}

The device physical port model file describes the network connection in the process layer network. The model file is organized in a syntax format similar to that of SCL. Model files to process layer network subnet as the basic unit. Each unit contains the subnet The IED device, the port number of the switch, and the physical cable (cable) connection identification. The physical port model can be saved as a XML file individually, or embedded in the "Communication" node of the configuration description file (SCD) of the substation.

The sub network of the process network in smart substation is defined by "SubNetwork" element, and the attribute values include "name", "type" and "desc". The device physical description of each subnet is defined by the "ConnectedAP" element. The attribute values for the ConnectedAP element include "iedName" and "apName"'". When the device is a switch, it should also include the data channel ID property "dpid"'. The ConnectedAP element contains a number of "PhysConn" elements that describe the physical ports of device access points. When the type attribute of the "PhysConn" element is "Connection", the first physical network port is defined, and the "RedConn" is defined as other redundant physical connection network ports. When redundant connections or multiple connections are used, PhysConn elements can be repeated, but the "type" attribute should be "RedConn".
The $<$ P type $=$ "Cable" $>$ element indicates the physical cable (or cable) connection identifier that is used to connect the two access points.

The $<$ P type $=$ "Port" $>$ element indicates the port number.

\section{B. Build Model Data Structure}

Xerces C++ DOM is used to parse the physical port model file and establish the following structure and mapping relation in memory.

1) Device port structure. The structure is the smallest unit for storing model files in memory, in which the device name and port number information are recorded.

Create a structure based on each port of each device. As shown below, the structure name is IED, which contains two members, where name is the device name and port is the device port number.

2) Link structure. The structure defines the link related information, named Link. Contains 3 members, where cable is the identity of the link, the device is the IED structure of the device, and the switch is the switching device IED structure. Through this structure, you can know which link of the switch is connected to the device through which link.

3) Subnet link mapping relation. The mapping relationship is a "one-to-many" associated storage container, which describes the correspondence between the subnet of the process layer network and the link structure Link. The key of the container is the name property of the subnet, and the value is the Link structure in the subnet.

The organizational structure of the associative storage container is shown in the following figure:

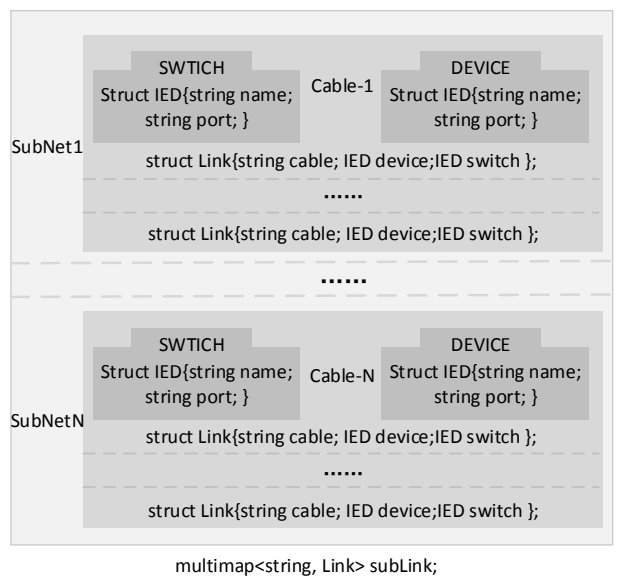

FIGURE II. ASSOCIATED CONTAINER SCHEMATIC DIAGRAM

The flow of the associated container is shown below: 


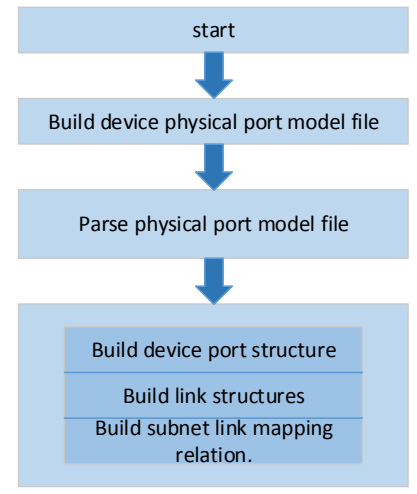

FIGURE III. GENERATE ASSOCIATIVE CONTAINER FLOW CHART

\section{Process Layer Network Automatic Configuration}

The process layer network is automatically configured by configuring the group table and flow table implementation in SDN technology. According to the "subnet-link" mapping relationship obtained above, extracte all the link structures in the subnet, and extracte the name and port number of the switch in the link structure. Configure the port number as a multicast group. The id of the multicast group corresponds to the subnet. In a multicast group, a multicast member sends a message, and all other multicast members are set to receive. The multicast type is set to "ALL". The action bucket should contain the "actions" action type, "type" in "actions" "Set to" OUTPUT "," port "is the port number. The relationship between the model port number and the action bucket is shown below:

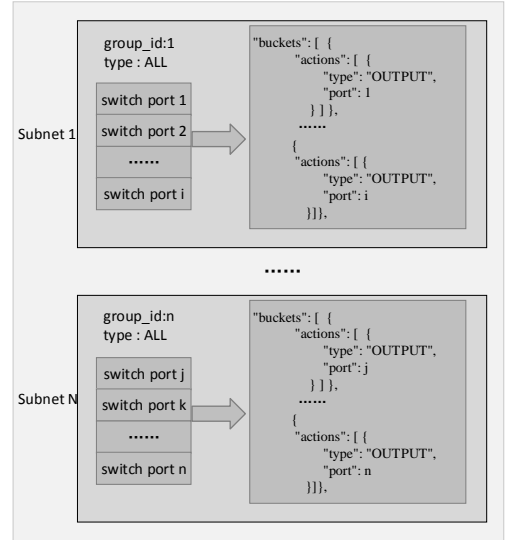

FIGURE IV. PORT AND ACTION BUCKET RELATION GRAPH

Group table calls are implemented by the flow table, which consists of several stream table entries. Add the flow table entry in the order of the port number, where "in_port" is the logical port number of the port, the group table is invoked through GROUP, and group_id is the group table ID for the subnet where the port is located. Take the switch port 1 as an example, the structure of the flow table entry is as follows:

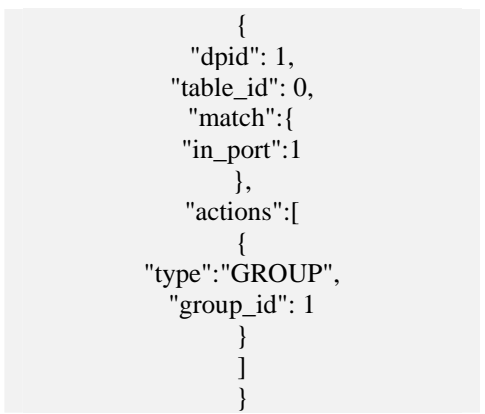

IV. SIMULATION EXPERIMENT

\section{A. Introduction to Mininet}

Mininet is a lightweight software definition network development and testing platform, based on OpenFlow, Open vSwitch various protocols for development and validation. Including the following main features: support for Open Flow, Open vSwitch and other software to define network protocols and components; support system-level restore test; support complex topology, custom topology; provide Python API; highly scalable, support more than 4096 host network Structure and so on.

\section{B. Simulation Experiment Based on Mininet}

1) Simulation environment

The physical environment is built on the virtual machine "VMware Workstation", its memory is 4GB, and the hard disk is 30GB. The software environment is shown in the following table:

TABLE I. SOFTWARE ENVIRONMENT

\begin{tabular}{ccc}
\hline Software & Version & function \\
\hline Ubuntu & 14.04 & $\begin{array}{c}\text { operating system } \\
\text { SDN network simulation } \\
\text { Mininet }\end{array}$ \\
platform \\
Ryu & 2.2 .1 & SDN controller \\
Open vSwitch & 2.4 .0 & SDN virtual switch \\
\hline
\end{tabular}

2) Establish a network topology

Based on Mininet, the network topology shown in Figure 413 is established. Which contains an SDN switch and 10 IED devices, 10 devices are connected with the 10 ports of the switch, the physical port model file in accordance with this topology to establish, of which 1 to 4 ports connected to the subnet "GOOSE" network, $6 \sim 9$ port Connect the subnet "SV" network, 5 10 port for the spare port, temporarily not involved in data transmission.

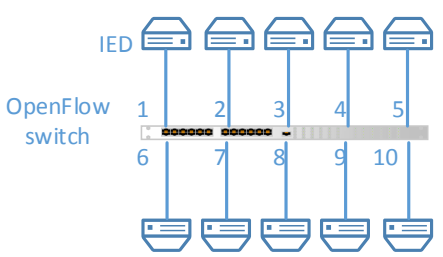

FIGURE V. NETWORK TOPOLOGY MAP 
3) Build device physical port model file

Build the device physical port model file, and part of the code is as follows:

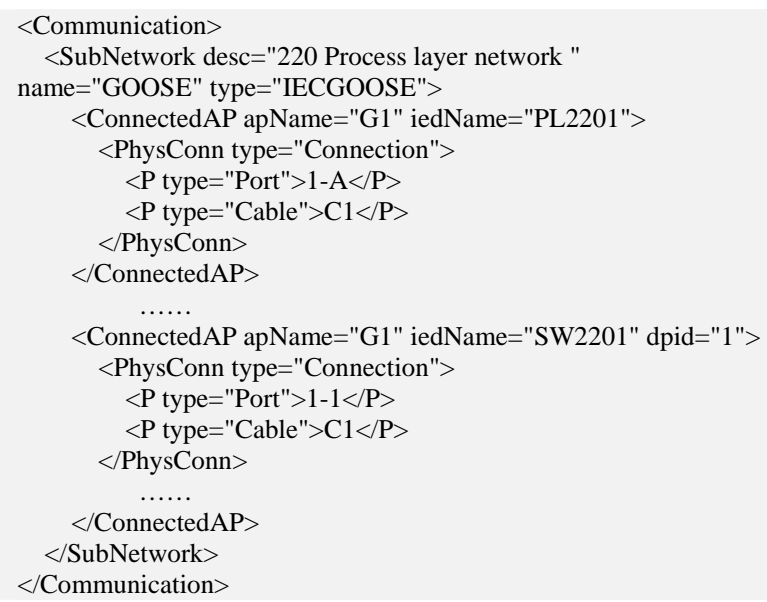

\section{4) Load the physical port model file}

Parse the model file, generate the group table and flow table and send it to the switch. The link and device port mapping is shown in the following figure:

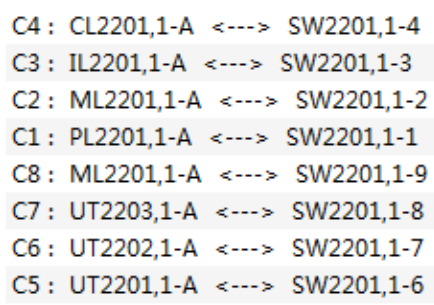

FIGURE VI. LINK AND DEVICE PORT MAPPING DIAGRAM

\section{(5) Experimental results}

The operation of pingall on the topology, operation results as shown below, it can be seen that the 1 4 device can be forwarding packets belonging to the same multicast group, 6 9 equipment can be forwarding packets belonging to the same multicast group.Devices 5 and 10 are backup devices, so port 5 and 10 do not send any configuration of flow tables and groups, therefore, packets cannot be forwarded with other ports. The experimental results are consistent with the configuration of the process layer network in the device physical port model.

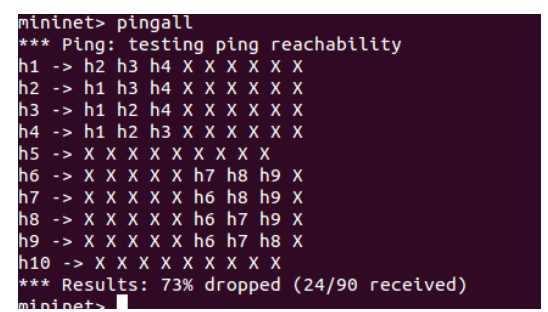

FIGURE VII. SIMULATION RESULTS

In this paper, a smart substation process layer network automatic configuration technology is proposed. The technology is used to automate the process layer network configuration by establishing a physical port model of the device and combining with SDN technology. Through simulation experiments, the feasibility of this technology is verified, which reduces the difficulty of network configuration of process layer, improves the efficiency of configuration work in process network of Smart Substation and reduces the workload of engineers. At the same time, by extending the physical port model of the device, the port traffic and QoS policy of the switch in the process layer network can be configured further. By extending the model and increasing the port information of the substation control layer network, the automatic control of the substation control layer network can be carried out automatically.

\section{REFERENCES}

[1] ZHU Lin, WANG Pengyuan, SHI Dongyuan. "Status monitoring information model and configuration description of communication network in smart sbustation,” J. Automation of Electric Power Systems, 2013, 37(11), pp. 87-92.

[2] LI Jungang, SONG Xiaohui, DI Junfeng, et al. "Communication network redundancy design of smart substation based on IEC 62439-3,” J. Automation of Electric Power Systems, 2011, 35(10),pp. 70-73.

[3] XIN Peizhe, YAN Peili, XIAO Zhihong, et al. "Application of communication network technology in new generation smart substation,” J. Electric Power Construction, 2013, 34(7), pp. 17-23.

[4] FAN Chen, NI Yimin, DOU Renhui, et al. "Analysis of network scheme for process layer in generation smart substation," J. Automation of Electric Power Systems, 2011, 35(18), pp. 67-71.

[5] XU Yong, LU Yujun, ZHANG Lei. "Design and realization of switches monitoring in smart substation ,” J. Jiangsu Electrical Engineering, 2014, 33(2), pp. 52-55..

[6] CHEN Honhui, WANG Haizhu. "Methods of VLAN configuration of smart substation based on SCD analysis," J. Electric Power Information and Communication Technology, 2014, 12(9), pp. 50-56..

[7] HUANG Xin, CHANG Wenzhi, WANG Feng, et al. "An automatic configuration method for process-level network in smart substation,” J. Electric Power Information and Communication Technology, 2015, 13(4), pp. 22-25..

[8] LIU Lian, HAN Shuai, LV Wengang, et al. "Research on architecture and development of software-defined networking," J. Information Technology \& Standardization, 2015 (9), pp. 61-64.

[9] LIU Lin, ZHOU Jiantao. "Review for research of control plane in software-defined network,” J. Computer Science, 2017,44 (2), pp. 75-81.

[10] ZHANG Tuanli, LV Guanghong, YANG Peilin. "Reliability of OpenFlow-based SDN,” J. Electronic Science and Technology, 2016, 29(2), pp. 177-181.

[11] ZHANG Junshuai, YANG Hao. "Design and implementation of openflow switch flow table forwarding," J. Journal of China University of Metrology, 2015, 26(3),pp. 316-323. 